\title{
Etude expérimentale de l'influence d'un champ de pression fluctuant sur l'apparition de la cavitation dans un venturi
}

\author{
J.Y. Billard \\ Laboratoire d'Hydrodynamique \\ de l'Ecole Navale \\ 29240 Brest Naval
}

\author{
D.H. Fruman \\ Groupe Phénomènes d'Interface \\ 91120 Palaiseau
}

\section{Introduction}

La cavitation apparaît au sein d'un écoulement lorsque la pression locale diminue et devient égale à une pression critique qui dépend du rayon des germes, du comportement thermodynamique du gaz inclus et de la façon dont s'accomplit la diminution de pression. Si cette dernière s'effectue sur un temps caractéristique supérieur à celui des oscillations propres du germe à la pression d'équilibre la formulation classique [CHAHINE et DARROZES, 1983] conduit au critère quasi statique auquel ne peut être associé qu'un comportement isotherme du gaz inclus. Si, par contre, la relation des temps est inversée, saut de pression par exemple, on est conduit [MАтsumoto et BEYLICH, 1985, Dugué et al., 1992] à un critère dynamique de cavitation qui fournit dans le cas isotherme une pression critique plus élevée que celle donnée par le critère quasi statique. Cependant, dans la situation dynamique le comportement du gaz inclus ne reste pas forcément isotherme. L'augmentation du coefficient polytropique, $k$, entraîne une diminution de la valeur de la pression critique dynamique qui reste supérieure $(k<1,25)$ ou devient inférieure $(k>1,25)$ à la pression critique quasi statique.

Une situation de croissance dynamique peut aussi se produire quand l'on superpose à un saut de pression une fluctuation harmonique de faible amplitude par rapport au saut et telle que le minimum reste toujours supérieur à la pression critique quasi-statique. L'intégration numérique de l'équation de Rayleigh-Plesset, pour un comportement isotherme des gaz inclus [BILLARD, 1991], montre que des conditions propices à la cavitation des germes apparaissent si la fréquence de la perturbation est de l'ordre de grandeur de la fréquence des oscillations non linéaires du germe soumis à la seule variation de la pression moyenne. Cette loi de pression correspond à une simulation simplifiée des conditions qui peuvent exister dans un écoulement turbulent. Le type de comportement ainsi mis en évidence a été appelé croissance asymptotique prématurée.

Pour offrir un support expérimental à ces résultats, l'apparition de la cavitation a été étudiée dans une boucle d'essais qui comporte une veine de type venturi, de $5 \mathrm{~cm}$

\section{Effect of a fluctuating pressure field on the behaviour of a cavitation bubble}

It is well known that a nucleus subject to a quasi-steady mean pressure drop can either oscillate or asymptotically grow depending the final pressure is above or blow the critical one. Recent investigations have shown that dynamically driven asymptotic growth conditions are associated to sudden mean pressure drops and to small amplitude pressure fluctuations superposed to a mean pressure larger than the quasi-steady critical pressure. In order to provide experimental support to the later results, tests were conducted to determine the effect of the modification of the upstream turbulence on the critical cavitation conditions in a venturi. The results show that cavitation is delayed when turbulence fluctuation intensities increase. A simple analysis associates this effect to the adiabatic behaviour of the gas within the cavity. 


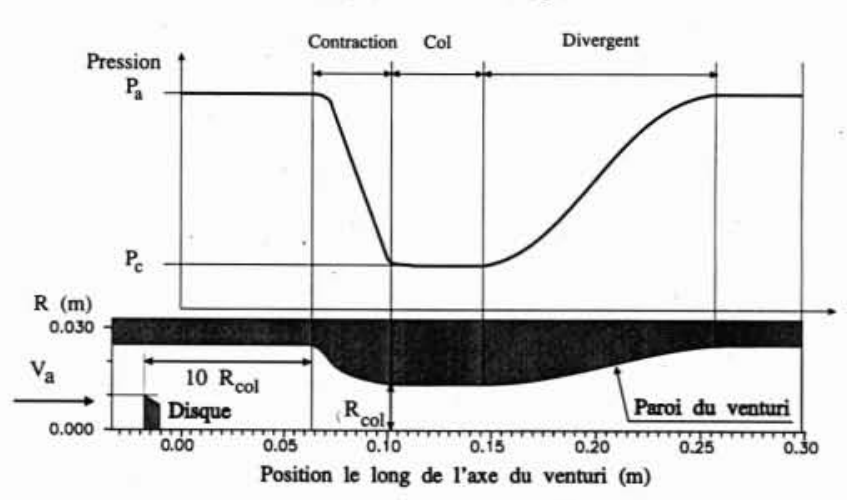

1. Schéma du dispositif expérimental et de la loi de pression associée.

de diamètre amont et de $2,6 \mathrm{~cm}$ de diamètre au col, dans laquelle transitent les germes de cavitation. Au champ de pression moyenne dans la veine on superpose une perturbation en intercalant un obstacle, constitué d'un disque à bord vifs de $2 \mathrm{~cm}$ de diamètre et de $5 \mathrm{~mm}$ d'épaisseur, placé à 5 diamètres au col en amont de l'entrée du convergent. Ce dispositif expérimental est présenté sur la figure 1. De manière à individualiser clairement l'effet de l'obstacle, celui-ci fait partie d'un montage mécanique permettant son introduction dans la canalisation amont sans modifier en quoi que ce soit ni les conditions d'écoulement ni les caractéristiques du fluide en recirculation. Une instrumentation adaptée, constituée essentiellement d'un détecteur du bruit émis, de capteurs de pression et de moyens de visualisation des structures cavitantes a été mise en place. La prise de pression absolue se situe à $7 \mathrm{~cm}$ en amont du disque et une prise analogue placée à $3,5 \mathrm{~cm}$ en aval permet de mesurer la perte de pression au passage du disque. Du fait du caractère aléatoire des phénomènes étudiés, un soin tout particulier a été apporté à l'acquisition et au traitement des données de manière à assurer leur validité statistique. Des mesures de vitesses et de taux de turbulence ont été effectuées par vélocimétrie laser dans des sections particulièrement intéressantes du venturi.

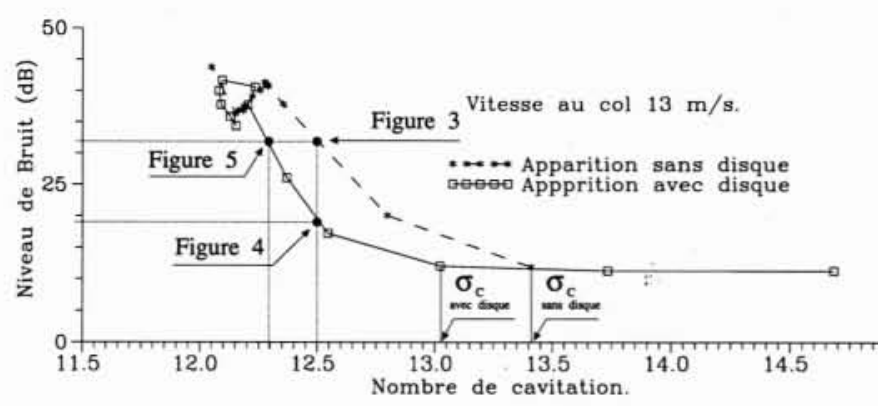

2. Comparaison entre les niveaux de bruit lors d'apparitions de cavitation avec et sans générateur de turbulence.

\section{Mesures et résultats}

Le nombre de cavitation est défini habituellement par:

$$
\sigma=\frac{P_{a}-P_{v}}{\frac{1}{2} \rho V_{a}^{2}}
$$

où $P_{v}$ est la pression de vapeur calculée à la température de l'essai, $\rho$ la densité de l'eau de circulation, $P_{a}$ la pression absolue au niveau de la prise de pression située en aval du disque et $V_{a}$ la vitesse dans la section amont du venturi.

Le niveau de bruit, valeur quadratique moyenne du signal sonore rapporté au niveau de bruit de fond de l'installation et exprimé en décibels, est reporté sur la figure 2 en fonction de $\sigma$, pour l'une des nombreuses expériences conduites. La présence de l'obstacle donne lieu à l'augmentation du niveau de bruit pour une valeur plus faible de $\sigma$. C'est donc un retard à l'apparition de la cavitation qui est mis en évidence. Ce résultat a été confirmé pour toutes les vitesses débitantes essayées ainsi que pour la disparition de la cavitation. De manière à mieux saisir les changements qualitatifs associés à ce retard à la cavitation, des prises de vues au col du venturi ont été effectuées pour différentes conditions d'écoulement.

Des photographies montrant la morphologie des inclusions gazeuses qui apparaissent au col sont présentées sur la figure 3 (sans disque) et sur les figures 4 et 5 (avec disque).

On note que pour des vitesses strictement identiques :

i) en l'absence de disque et pour une cavitation développée $(\sigma=12,5)$, figure 3 , les bulles sont assez nombreuses, isolées les unes des autres et ont une forme à peu près sphérique. Le niveau de bruit correspondant est de $32 \mathrm{~dB}$ (voir fig. 2). La présence de quelques inclusions gazeuses non sphériques résulte des forts gradients de vitesses au voisinage de la paroi et de la prise de pression pariétale qui crée une amorce locale de cavitation;

ii) avec le disque et pour la même valeur de $\sigma$, figure 4 , la croissance des inclusions gazeuses est considérablement réduite. Par ailleurs, le niveau de bruit a largement décru puisqu'il n'est plus que de $19 \mathrm{~dB}$. On note que la cavitation attachée à la prise de pression placée au col du venturi a fortement diminué ;

iii) si, toujours en présence de l'obstacle, on modifie la pression amont pour revenir à un niveau de bruit de $32 \mathrm{~dB}$, on atteint des nombres de cavitation plus faibles $(\sigma=12,3)$ et l'on constate la formation d'inclusions gazeuses plus grosses et de formes très torturées, figure 5 . Même dans ces conditions, la cavitation attachée à la prise de pression ne retrouve pas l'ampleur qu'elle avait sur la photographie de la figure 3 .

Le retard à la cavitation et la modification de la morphologie des inclusions semblent être directement associés à la modification de la turbulence due à la présence du disque. En effet, les mesures par anémométrie laser dans le col, pour des conditions de débit identiques, ont montré que le profil des vitesses hors couche limite n'est pas modifié alors que le taux de turbulence, là où les vitesses axiales restent pratiquement constantes, passe de 1,4 à $3,7 \%$, figure 6 . 


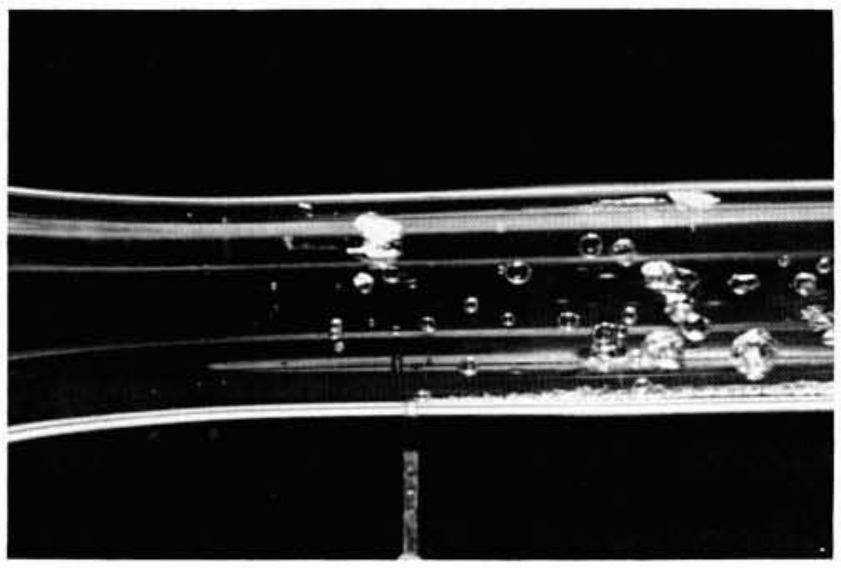

3. Cavitation dans le venturi sans disque. Conditions: $V_{\text {col }}=13 \mathrm{~m} / \mathrm{s} \quad(\operatorname{Re}=340000), \quad \sigma=12,5, \quad$ Niveau de bruit $=32 \mathrm{~dB}$

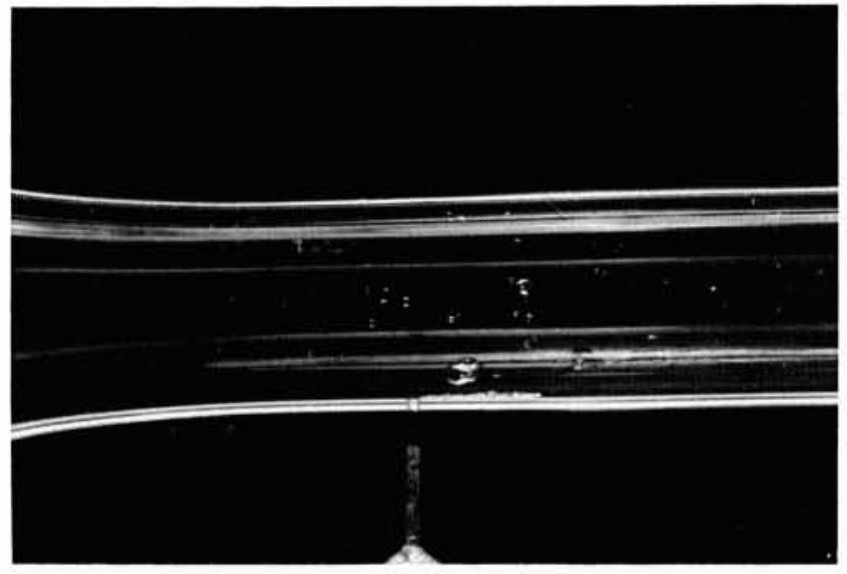

4. Cavitation dans le venturi avec disque. Conditions : $V_{\mathrm{col}}=13 \mathrm{~m} / \mathrm{s} \quad(\operatorname{Re}=340000), \quad \sigma=12,5, \quad$ Niveau de bruit $=19 \mathrm{~dB}$.

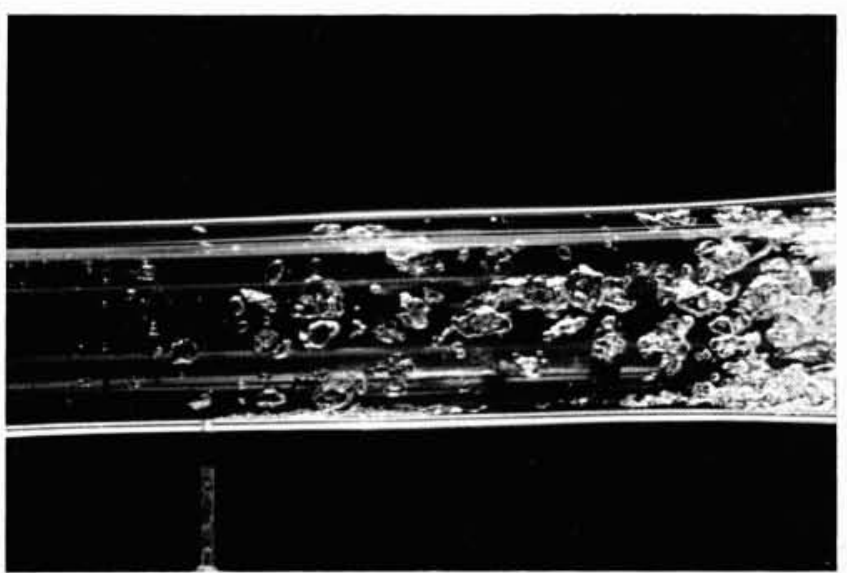

5. Cavitation dans le venturi avec disque. Conditions: $V_{\text {col }}=13 \mathrm{~m} / \mathrm{s} \quad(\operatorname{Re}=340000), \quad \sigma=12,3, \quad$ Niveau de bruit $=32 \mathrm{~dB}$.

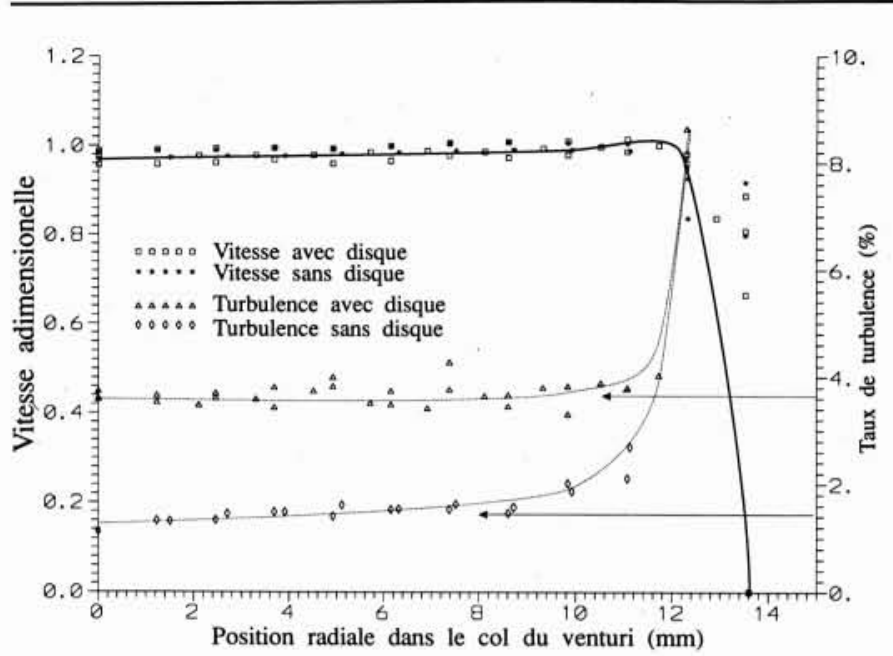

6. Profil des vitesses et des taux de turbulence dans la section du $\mathrm{col}$.

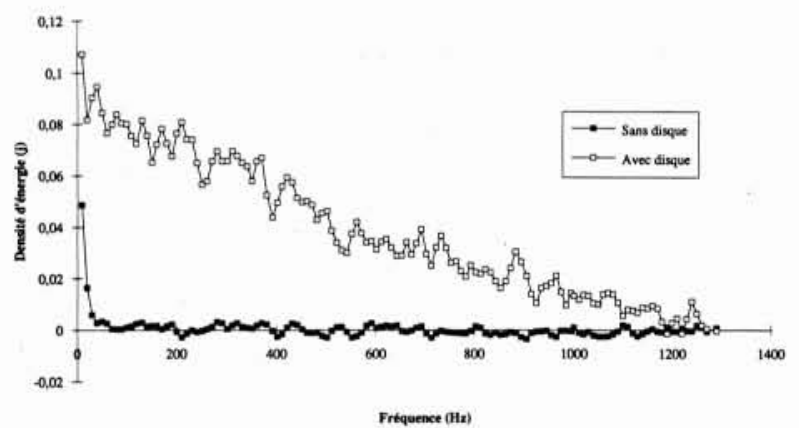

7. Comparaison des spectres de turbulence au col et sur l' axe du venturi.

Bien que les fluctuations aléatoires dues à la turbulence soient assez éloignées de la perturbation harmonique utilisée dans l'étude numérique [BILLARD, 1991], on peut tenter une interprétation des résultats en procédant à l'analyse spectrale du signal de vitesse obtenu au col du venturi sur l'axe de la conduite. La figure 7 présente deux spectres mesurés dans les mêmes conditions de fonctionnement de l'installation avec et sans disque. En l'absence de disque, les seules composantes significatives sont celles à très basse fréquence $(<100 \mathrm{~Hz})$ qui donc agissent de façon quasi statique sur l'évolution des germes. L'introduction du disque entraîne l'apparition de composantes à des fréquences plus élevées, jusqu'à $1200 \mathrm{~Hz}$, qui conduisent à un comportement dynamique des germes. En effet, la fréquence des oscillations non linéaires d'un germe de $10 \mu \mathrm{m}$ de rayon initial soumis à une chute allant de la pression atmosphérique à une pression de $1500 \mathrm{~Pa}$ est de $10 \mathrm{kHz}$; presqu'un ordre de grandeur supérieur. Cependant, BILlaRd [1991] a montré que les effets dynamiques se font sentir même pour des fréquences d'excitation inférieures à la fréquence propre non linéaire. 


\section{Discussion des résultats}

Les différents essais réalisés mettent en évidence l'existence d'un retard à la cavitation lorsque l'on introduit un générateur de turbulence en amont du venturi. On peut envisager, comme le suggère le critère dynamique, que le retard constaté expérimentalement soit associé à la modification du comportement thermodynamique du gaz inclus. Pour étayer cette hypothèse on procède comme suit :

i) à partir du $\sigma_{c}$ d'apparition sans disque (fig. 2) et de l'équation de Bernouilli appliquée entre la section amont et le col (en négligeant les pertes de charge) on calcule la pression critique au col. En tenant compte des caractéristiques du spectre de turbulence sans disque, on peut supposer que le processus donnant lieu à l'apparition de la cavitation peut être décrit par le critère quasi statique, et l'on peut alors calculer le rayon des germes le plus probable, $r_{0}$, avec la formule usuelle [DARROZES et Chahine, 1983]. Dans le cas qui nous occupe il est de $8 \mu \mathrm{m}$ à la pression atmosphérique $P_{\infty 0}$;

ii) on procède à un calcul en tout point similaire en utilisant le $\sigma_{c}$ d'apparition avec disque de manière à obtenir la pression critique au col qui est maintenant assimilée à celle correspondant à une croissance dynamique dont le coefficient polytropique reste inconnu. Pour le déterminer on construit, avec cette pression critique et le diamètre des germes le plus probable, le nombre $w_{c d}^{*}=\frac{P_{\infty}-P_{v}}{2 \gamma} r_{0}$, où $P_{\infty}$ est la pression critique calculée et $\gamma$ la tension superficielle. On calcule alors $w_{0}=$ $\frac{P_{\infty 0}-P_{v}}{2 \gamma} r_{0}$ et l'on cherche à l'aide des tables fournies dans DuguÉ et al., [1992], pour quelle valeur du coefficient polytropique sont associées les valeurs $w_{c d}^{*}$ et $w_{0}$. La valeur du coefficient polytropique obtenue, 1,3 , est physiquement admissible et tout à fait apte à décrire un phénomène pour lequel les équilibres thermiques n'ont pas le temps d'être atteints.

Quoique l'utilisation d'un coefficient polytropique pour décrire le comportement thermique du gaz inclus ne donne qu'une description approchée de la physique du phénomène, la tendance doit en principe rester la même
[MATSumoto et Beylich, 1985] si l'on procède à un calcul complet en tenant compte de l'équation de l'énergie.

\section{Conclusions}

Les caractéristiques de l'écoulement au col d'un venturi suivant qu'un obstacle générateur de turbulence soit ou non placé en amont de celui-ci ont été déterminées par anémométrie laser. On a montré que le profil des vitesses n'était pas altéré par la présence de l'obstacle tandis que le taux de turbulence augmentait d'un facteur voisin de $2,6(1,4$ à $3,7 \%)$. l'analyse spectrale des vitesses fluctuantes montre par ailleurs un accroissement de la fréquence de coupure supérieure. La bande de fréquence capable d'exciter les germes d'une taille voisine de $10 \mu \mathrm{m}$ de rayon est alors porteuse d'une énergie suffisante pour pouvoir les activer par un processus dynamique. Ces modifications de l'écoulement au col semblent être responsables d'un retard à l'apparition de la cavitation dans la plage -de paramètres de fonctionnement utilisée.

Ces résultats peuvent être associés à ceux issus de recherches récentes concernant les situations de croissance asymptotique sous l'effet de changements brutaux de la pression moyenne et conduisant à l'établissement d'un critère dynamique. En effet, une analyse simple des résultats expérimentaux a permis d'imputer le retard à la cavitation au comportement adiabatique des gaz inclus. Cette recherche doit se poursuivre par l'étude de fluctuations de pression monochromatiques sur les conditions d'apparition de la cavitation.

\section{Remerciements}

Ce travail a été financé par la Direction de Recherches, Etudes et Techniques (D.R.E.T.) dans le cadre du Contrat $n^{\circ} 88 / 1040$. Monsieur P. Galivel, Maître de Conférences à l'IUT de Quimper, a activement participé à la réalisation d'une partie des montages et des expériences. Qu'il trouve ici l'expression de nos remerciements sincères.

\section{Références}

Chahine G.L. et DARRozes J.S., 1983. - « Les recherches sur le phénomène de cavitation effectuées à l'Ecole Nationale Supérieure de Techniques Avancées ». Extrait de «Sciences et Techniques de l'Armement " mémorial de l'Artillerie française, $1^{\text {er }}$ fascicule 1983.

Matsumoto Y. et BeYLich A.E., 1985. - «Influence of Homogeneous Condensation inside a Small Gas Bubble on its Pressure Response ». Journal of Fluids Engineering, vol. 107, June 1985, pp. 281-286.

BILlARD J.Y., 1991. - "Influence d'un champ de pression fluctuant sur la croissance et le collapse des cavités». Thèse de doctorat de l'université Paris VI.

Dugue C., Fruman D.H., Billard J.Y. et Cerrutti Ph., 1992. - « Dynamic Criterion for Cavitation of Bubbles ", vol. 114, june 1992, pp. 250-254. Journal of Fluids Engineering. 